\title{
Prognostic value of genomic alterations in head and neck squamous cell carcinoma detected by comparative genomic hybridisation
}

\author{
JNE Ashman', HS Patmore', LT Condon', L Cawkwell'2, ND Stafford' and J Greenman ${ }^{*, 3}$ \\ ${ }^{\prime}$ Academic Departments of Otolaryngology and Head and Neck Surgery, University of Hull, Hull HU6 7RX, UK; ${ }^{2}$ Academic Department of Oncology, \\ University of Hull, Hull HU6 7RX, UK; ${ }^{3}$ Academic Surgical Unit (JG), University of Hull, Hull HU6 7RX, UK
}

\begin{abstract}
A total of 45 primary head and neck squamous cell carcinomas were analysed by comparative genomic hybridisation to identify regions of chromosomal deletion and gain. Multiple regions of copy number aberration were identified including gains affecting chromosomes 3q, 8q, 5p, 7q, I 2p and I I q and deletion of material from chromosomes 3p, I I q, 4p, 5q, 8p, I0q, I 3q and 2I. KaplanMeier survival analysis revealed significant correlations between gain of 3q25-27 and deletion of 22q with reduced disease-specific survival. In addition, gain of $17 q$ and $20 q$, deletion of $19 p$ and $22 q$ and amplification of $1 / q \mid 3$ were significantly associated with reduced disease-free survival. A Cox proportional hazards regression model identified deletion of 22q as an independent prognostic marker. The data presented here provide further evidence that the creation of a genetically based tumour classification system will soon be possible, complementing current histopathological characterisation.

British Journal of Cancer (2003) 89, 864-869. doi: I 0.1038/sj.bjc.660I I99 www.bjcancer.com
\end{abstract}

(C) 2003 Cancer Research UK

Keywords: head and neck squamous cell carcinoma; comparative genomic hybridisation; survival

Head and neck squamous cell carcinoma (HNSCC) is the fifth most common cancer worldwide and contributes significantly to the morbidity and mortality associated with human cancer. Despite numerous advances in treatment the long-term survival rates for HNSCC have changed little over the last 20 years (Jemal et al, 2003). Currently, the management of HNSCC is based on the assessment of a variety of clinical and pathological parameters, of which nodal status conveys the strongest prognostic information (O’Brien et al, 1986). However, in many instances, these factors fail to predict accurately the clinical behaviour of an individual patient's tumour. A greater understanding of the molecular basis of HNSCC will hopefully allow the biological properties of an individual patient's tumour, including metastatic potential, response to therapy and likelihood of recurrence, to be more precisely determined.

Definition of the genetic changes underlying HNSCC is underway but remains incomplete. Classical cytogenetic analysis of HNSCC tumours has revealed multiple chromosomal abnormalities and highly complex karyotypes. The most widely reported aberration demonstrating a correlation with clinical outcome is amplification of chromosome 11q13. Both the amplification of this band and overexpression of the cyclin D1 oncogene have been repeatedly associated with poor prognosis (Akervall et al, 1995; Meredith et al, 1995). Many of the limitations of conventional

*Correspondence: Dr J Greenman, Medical Research Laboratory, Wolfson Building, University of Hull, Cottingham Road, Hull HU6 7RX, UK; E-mail: j.greenman@hull.ac.uk

Received II February 2003; revised 10 June 2003; accepted 24 June 2003 cytogenetic analysis have been overcome with the development of molecular techniques including FISH and comparative genomic hybridisation (CGH). Comparative genomic hybridisation is a powerful technique that screens the entire tumour genome for DNA sequence copy number alterations. Comparative genomic hybridisation has previously been applied in several HNSCC studies and has demonstrated a nonrandom pattern of genomic aberrations including deletions of material from $3 p, 4 q, 5 q, 9 p, 18 q$ and gains involving $3 \mathrm{q}, 5 \mathrm{p}, 7 \mathrm{p}, 8 \mathrm{q}, 11 \mathrm{q}, 17 \mathrm{q}$ and $20 \mathrm{q}$ (reviewed in Struski et al, 2002).

In this study, we have applied CGH to HNSCC patients in order to characterise the chromosomal imbalances underlying this disease and evaluated the prognostic significance of the aberrations identified.

\section{METHODS}

\section{Patients}

Informed consent was obtained from all patients and local ethical approval granted for the study. In total, 53 consecutive patients undergoing surgery for a single primary HNSCC between April 1996 and November 2001 were included in this study. Biopsy-sized specimens were obtained at the time of surgical resection, snap frozen in theatre and stored at $-80^{\circ} \mathrm{C}$ until analysis. Specimens were taken immediately adjacent to specimen sent for histopathological assessment and all samples were analysed by a single pathologist. Several patients were excluded from the study cohort for the following reasons: only specimens containing $>70 \%$ malignant cells upon pathological examination were included, as 
Table I Clinicopathological details of the 45 HNSCC patients included in this study

\begin{tabular}{|c|c|c|c|}
\hline & Total & Alive & Dead of disease \\
\hline No. of patients & 45 & 28 & 17 \\
\hline Male & 35 & 22 & 13 \\
\hline Female & 10 & 6 & 4 \\
\hline \multicolumn{4}{|l|}{ Tumour site } \\
\hline Larynx & 21 & 15 & 6 \\
\hline Hypopharynx & 13 & 9 & 4 \\
\hline Oropharynx/oral cavity & 10 & 4 & 6 \\
\hline Parotid & । & 0 & । \\
\hline \multicolumn{4}{|l|}{ Stage } \\
\hline TI & I & I & 0 \\
\hline $\mathrm{T} 2$ & 12 & 8 & 4 \\
\hline T3 & 9 & 8 & i \\
\hline T4 & 23 & 11 & 12 \\
\hline No & 15 & 13 & 2 \\
\hline $\mathrm{N}+$ & 30 & 15 & 15 \\
\hline $\mathrm{NI}$ & 8 & 5 & 3 \\
\hline N2 & 20 & 10 & 10 \\
\hline N3 & 2 & 0 & 2 \\
\hline \multicolumn{4}{|l|}{ Differentiation } \\
\hline Well & 3 & 2 & । \\
\hline Moderate & 18 & 11 & 7 \\
\hline Poor & 22 & 14 & 8 \\
\hline Undifferentiated & 2 & I & 1 \\
\hline
\end{tabular}

$\mathrm{N} 0=$ no nodal involvement; $\mathrm{N}+=$ with nodal involvement

CGH analysis requires that the tumour cells constitute the majority of the tissue specimen ( $n=5$ excluded). Patients who received radiotherapy prior to surgical resection were excluded from the cohort ( $n=3$ excluded) to avoid the inclusion of specimens containing radiotherapy-induced genetic changes. None of the 53 patients had received preoperative chemotherapy. The clinicopathological details of the 45 patients analysed are detailed in Table 1.

\section{Comparative genomic hybridisation}

DNA was extracted from either serial $20 \mu \mathrm{m}$ cryostat sections or whole biopsy by proteinase $\mathrm{K}$ digestion followed by phenolchloroform extraction. DNA quality and purity were assessed by electrophoresis and spectrophotometry at $260 \mathrm{~nm}$, and CGH was performed essentially as described previously (Stafford et al, 1999). All CGH reagents were obtained from Abbott Laboratories Ltd (Maidenhead, UK). All experiments were performed in combination with both positive (DNA with known aberrations) and negative (normal: normal hybridisation) control experiments. Sex mismatching of test and reference DNA precluded the analysis of the sex chromosomes. Deletions and gains of DNA were identified whenever the CGH ratio profile exceeded thresholds established through normal:normal hybridisations ( 0.85 and 1.15 respectively).

\section{Statistical analysis}

Disease-specific survival time was calculated as the time between the date of tissue acquisition (surgical resection) and death. Only patients with cancer-specific death were included in the analysis and patients alive at the time of last follow-up were censored accordingly. Disease-free survival was defined as the time between tissue acquisition and evidence of disease recurrence. For purposes of statistical analysis local, regional or distant recurrence were grouped together as disease recurrence. Survival analysis was performed using the Kaplan-Meier method and the difference in survival curves was tested for statistical significance using the log rank test. Cox proportional hazard analysis was used to test variables identified by Kaplan-Meier analysis for independent prognostic significance. The $\chi^{2}$ test was used to evaluate relationships between categories. All statistical analysis was performed using the SPSS version 10 software package and values of $P<0.05$ were considered to be statistically significant.

\section{RESULTS}

\section{Overview of genomic changes in HNSCC}

Genetic aberrations were detected in all 45 specimens analysed and occurred across the entire genome. Figure 1 summarises all the chromosomal aberrations identified. Overall, CGH identified a complex pattern of aberrations; however, several frequent chromosomal loci of copy number change were seen. These included gains affecting chromosomes $3 \mathrm{q}, 8 \mathrm{q}, 5 \mathrm{p}, 7 \mathrm{q}, 12 \mathrm{p}$ and $11 \mathrm{q}$ and deletion of material from chromosomes $3 p, 11 q, 4 p, 5 q, 8 p$, $10 q, 13 q$ and 21 . Those occurring in $>20 \%$ of the cohort of tumours analysed are highlighted in Table 2 and are consistent with previously published CGH analyses of HNSCC. There was a mean of 18.8 aberrations per tumour (range 4-38), 9.4 deletions (range 1-23) and 9.5 gains (range 1-18). Amplifications (CGH ratio $>1.5$ ) were detected on $3 q$ (10 cases), $11 \mathrm{q}$ (four cases), $5 \mathrm{p}$ (two cases), $7 \mathrm{p}$ and $8 \mathrm{q}$ (one case each). There was no significant difference between the total number of copy number aberrations when patients were stratified by $\mathrm{T}$ stage, nodal involvement, tumour stage or differentiation. Only gain of $7 p$ and deletion of $9 q$ were significantly more frequent in patients with nodal involvement $(P=0.019$ and 0.012 respectively) and gain of $20 \mathrm{q}$ significantly associated with recurrent disease $(P=0.022)$.

\section{Clinical outcome}

Follow-up of the patients was continued until November 2002 and was available for all patients. The mean duration of follow-up was 35.0 months overall (95\% confidence interval (CI), 28.3-41.7). During this period, $37.8 \%$ of patients died from their disease (mean time to death 20.0 months, 95\% CI, 10.0-29.9) and $51.1 \%$ patients developed disease recurrence (including six local, 14 regional, one local and regional and two distant recurrences; mean time to disease recurrence 14.2 months, $95 \%$ CI, 8.6-19.7). Mean follow-up for patients remaining alive at time of analysis was 44.0 months (95\% CI, 36.9-51.2). The clinical outcome for patients with pathologically proven regional lymph node metastases was significantly worse than for patients with no nodal involvement with a mean survival of 43.9 months (95\% CI, 33.5-54.2) and 63.9 months (95\% CI, 51.1-75.4) respectively ( $P=0.024$; Figure $2 \mathrm{~A})$. When this analysis was extended to include patients who were not initially submitted for a neck dissection, but eventually presented with a neck recurrence ( $n=3$ additional tumours), this association was slightly stronger with a mean survival of 44.4 months (95\% CI, 34.6-54.1) for all patients with nodal involvement and 66.7 months (95\% CI, 55.5-78.0) for patients in which the regional lymph nodes remained disease-free through the course of followup $(P=0.021)$. The clinical and follow-up details of the eight patients excluded for reasons detailed in the Methods section did not differ significantly from the cohort of 45 patients for which genetic analysis was performed (data not shown).

\section{Correlation of CGH data with survival}

When disease-free survival was analysed, several aberrations demonstrated significant associations (Table 3). Gain of 3q25-27 and losses on $22 \mathrm{q}$ were the only aberrations significantly 

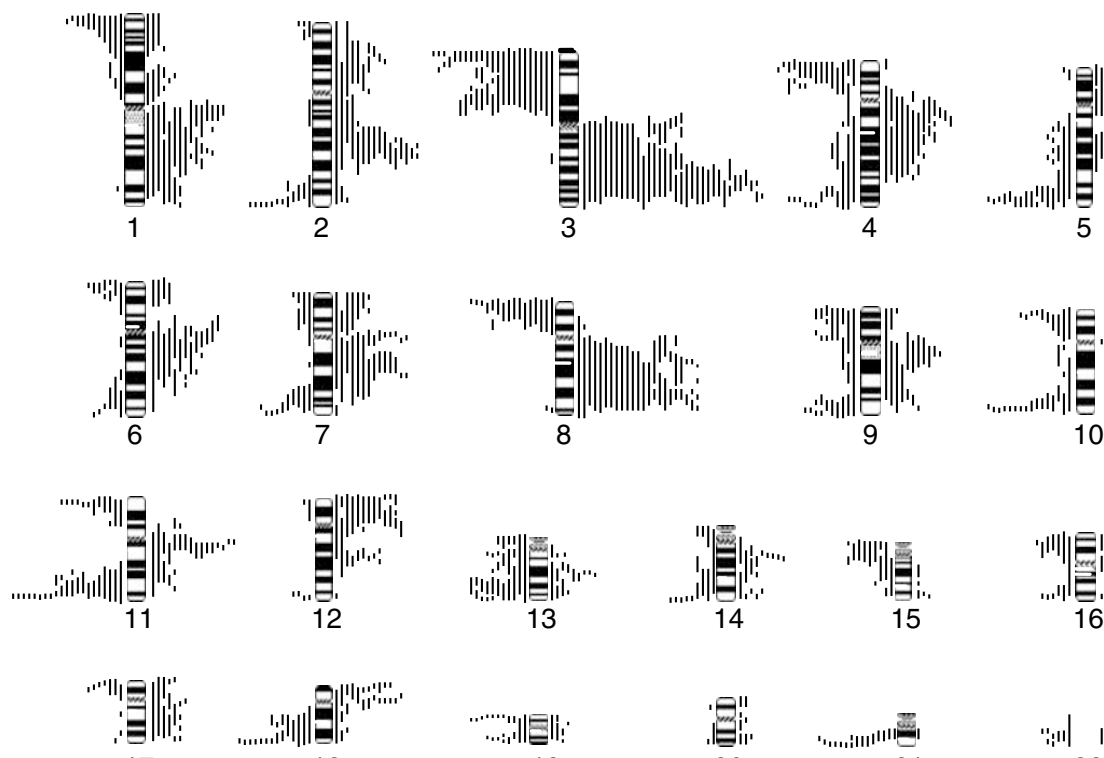

17

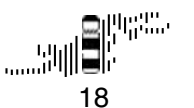

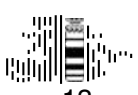

13

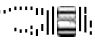

19
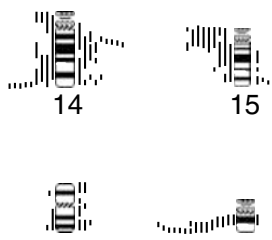
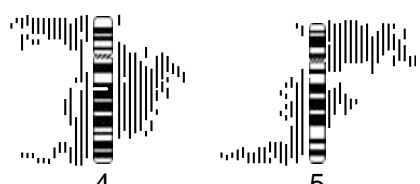

Figure I Summary of all chromosomal gains and losses identified in 45 primary HNSCC tumours by CGH. Lines to the left of the chromosome ideograms represent regions of deletion and lines to the right represent gains. The relative lengths of each line represents the size of the region of gain or loss. All CGH ratio deviations identified using the thresholds detailed in the methods were included. A few, particularly small, regions of copy number change below the accepted resolution of $\mathrm{CGH}(\sim 10 \mathrm{Mb})$ are present and care should be taken in interpreting these.

associated with disease-specific survival (Table 3, Figure 2). The mean survival for patients with gain of the chromosomal locus $3 \mathrm{q} 25$ - q27 was significantly reduced (44.9 months) when compared to those without (63.3 months) ( $P=0.04$; Figure $2 \mathrm{~B})$. Interestingly, this significance did not hold when patients with gains anywhere on $3 \mathrm{q}$ were compared with those without $(P=0.37$; Figure $2 \mathrm{C})$. The association of deletion of $22 \mathrm{q}$ with reduced survival was stronger $(P=0.01$; Figure 2D); however, only eight patients in the cohort demonstrated this deletion. Mean survival for patients with loss of $22 \mathrm{q}$ was 17.0 months compared with 54.9 months for those without. Cox proportional hazard analysis indicated that nodal status and deletion on $22 \mathrm{q}$ were independent prognostic variables (Table 4).

\section{DISCUSSION}

Comparative genomic hybridisation analysis of this cohort of tumours has provided further evidence for the importance of gain of material on $3 \mathrm{q}$ in the development of HNSCC. The gain of 3q25-27 was the most prevalent gain detected in this cohort of primary HNSCC. Kaplan-Meier survival analysis demonstrated a significant association of 3q25-27 gain with reduced overall survival; however, Cox regression analysis indicated this effect was not independent. Several other CGH studies have identified $3 \mathrm{q}$ as a consistent site of both gain of DNA and amplification (Bergamo et al, 2000; Bockmühl et al, 2000; Hashimoto et al, 2001; Hermsen et al, 2001; Singh et al, 2001). A similar association with survival has previously been identified by Bockmühl et al (2000). In their study of 113 primary HNSCC tumours, gain of material at 3q21q29 demonstrated a strong association $(P=0.006-\log$ rank test $)$ with a reduced overall survival. The significance of the difference between the region of chromosome $3 \mathrm{q}$ identified in this cohort $(3 q 25-27)$ and Bockmühl study (3q21-29) is unclear. Both studies involved heterogenous populations of HNSCC tumours, a major limitation of any HNSCC analysis; however, the overall sub-site distribution was similar. The observed difference may simply reflect the different methods of analysis employed. Bockmühl et al used a CGH analysis software package that allowed integrated statistical analysis of chromosomal alterations at each chromosomal band throughout the genome (at the ISCN 400 band resolution). In the cohort presented here, the region 3q25-27 represents the minimal region of overlap present in all tumours demonstrating a gain on $3 \mathrm{q} 2$.

The gain of $3 \mathrm{q}$ has been reported in numerous tumour types, most frequently in those of squamous origin, including HNSCC, squamous lung carcinomas and cervical carcinoma. (Chujo et al, 2002; Struski, 2002). In squamous cell carcinoma of the uterine cervix, the gain of $3 \mathrm{q}$ has been shown to define the transition from severe dysplasia to invasive carcinoma (Heselmeyer et al, 1996). The high frequency of $3 \mathrm{q}$ gains identified in the present study is consistent with $3 \mathrm{q}$ gain being an early event in HNSCC tumorigenesis. However, an early, essential, genetic requirement for tumorigenesis would be unlikely to carry prognostic value as demonstrated here and previously (Bockmühl et al, 2000). Recently, Hashimoto et al (2001) have further delineated the involvement of $3 \mathrm{q}$ gains in HNSCC tumorigenesis by demonstrating a correlation between amplification, as opposed to low level gain, of 3q26-qter and tumour progression. Hashimoto identified gain of 3q26-qter in $91 \%$ of tumours $(n=32)$, and amplification at a significantly higher frequency in T4 tumours $(70 \%)$ when compared with T2 and T3 tumours (both 27\%; $P<0.05$-Fisher's exact test). Using interphase FISH, Singh et al (2002) demonstrated a significant increase in 3q26-q27 amplification from normal mucosa $(3 \%)$, premalignant mucosa $(25 \%)$ to invasive cancer $(56 \% ; P<0.01)$. This study also showed a significant increase in tumour recurrence and cancer-related death when tumours were stratified by 3q26-q27 copy number (normal, low-level and highlevel $(>4 \times)$ amplification). It appears likely that multiple genes within the region of $3 q$ gain identified in this study are important for HNSCC progression. Alterations in a gene(s) early in HNSCC tumorigenesis may occur through low-level gains, while amplification of additional genes may occur later in the progression of HNSCC and perhaps characterise more aggressive tumours with a worse prognosis.

Several candidate genes have been localised to $3 \mathrm{q} 25-\mathrm{q} 27$ including the FGF12 growth factor, cyclin $L$ and PIK3CA (Redon et al, 2001, 2002). PIK3CA encodes the p110 catalytic subunit of 
phosphatidylinositol-3'-kinase, a critical component of many cell signalling pathways including those of EGF and PDGF (Volinia et al, 1994). Targeted FISH experiments using yeast artificial chromosomes (YACs) have demonstrated amplification of the PIK3CA gene in a panel of HNSCC cell lines (Singh et al, 2001).

Table 2 Frequencies of copy number imbalances identified in this study ( $>20 \%$ of cases), ranked according to the most frequently occurring

\begin{tabular}{|c|c|c|}
\hline Chromosome & Aberration & Frequency (\%) \\
\hline $3 q 25-q 27$ & Gain & 75.5 \\
\hline $3 q 21-q 24$ & Gain & 62.2 \\
\hline $8 q 22.2-$ qter & Gain & 55.6 \\
\hline 3p24-pter & Deletion & 51.1 \\
\hline I l q23. I -qter & Deletion & 48.9 \\
\hline $8 q 21.1-q 22.2$ & Gain & 48.9 \\
\hline 3qcen-q|3.3 & Gain & 46.7 \\
\hline $8 q c e n-q 21.1$ & Gain & 46.7 \\
\hline $5 p$ & Gain & 44.4 \\
\hline 4p|4.1-pter & Deletion & 42.2 \\
\hline $3 p|3-p| 3.3$ & Deletion & 40.0 \\
\hline $5 q 32-$ qter & Deletion & 37.8 \\
\hline $8 p 12$-pter & Deletion & 35.6 \\
\hline $7 p|2-q| 1.23$ & Gain & 35.6 \\
\hline $12 p$ & Gain & 35.6 \\
\hline||$q|3.1-q| 4.1$ & Gain & 35.5 \\
\hline 21 & Deletion & 35.5 \\
\hline $3 p 21.3-p 23$ & Deletion & 33.3 \\
\hline 10q24.I-qter & Deletion & 33.3 \\
\hline 13q22-qter & Deletion & 33.3 \\
\hline$|q 23-q 3|$ & Gain & 31.1 \\
\hline $4 q c e n-q 21.3$ & Gain & 31.1 \\
\hline $4 q 32-$ qter & Deletion & 28.9 \\
\hline $18 q 22-$ qter & Deletion & 28.9 \\
\hline $9 q 33$-qter & Deletion & 26.7 \\
\hline 6qcen-q16.I & Gain & 24.4 \\
\hline Ip35-pter & Deletion & 24.4 \\
\hline ||p|4-pter & Deletion & 24.4 \\
\hline $2 q 36$-qter & Deletion & 24.4 \\
\hline $18 q 11.2$ & Gain & 24.4 \\
\hline $9 q 13-q 22.2$ & Gain & 22.2 \\
\hline $18 p$ & Gain & 22.2 \\
\hline $19 p 13.2-$ pter & Deletion & 22.2 \\
\hline $7 q 31.3-$ qter & Deletion & 22.2 \\
\hline |Op|4-qter & Deletion & 22.2 \\
\hline $12 q 21.2-q 21.3$ & Gain & 22.2 \\
\hline$|4 q 13-q 2|$ & Gain & 20.0 \\
\hline $9 p 21-$ pter & Deletion & 20.0 \\
\hline $15 q|1.2-q| 5$ & Deletion & 20.0 \\
\hline
\end{tabular}

Increased copy number of the PIK3CA gene has also been identified in cervical cancer and ovarian cancer, and in the latter case has been shown to be associated with increased expression (Shayesteh et al, 1999; Ma et al, 2000).

The literature on the involvement of $22 \mathrm{q}$ in HNSCC progression is limited. Comparative genomic hybridisation analysis has demonstrated copy number changes in up to $50 \%$ of cases analysed with both deletion and, more commonly, gain of material from 22q having been reported. Preliminary LOH data have proposed that allelic imbalance on $22 \mathrm{q}$ is more frequent in laryngeal and oral tumours than other HNSCC (dos Reis et al, 2002). Comparative genomic hybridisation analysis of 22q, along with regions of $1 \mathrm{p}, 9 \mathrm{p}, 16$ and 19 can be problematic and should be interpreted with caution (Jeuken et al, 2002). Particularly stringent evaluation of simultaneous negative control experiments is required to identify experimental artefact within this region. All relevant negative control experiments were of a high quality and did not demonstrate false-positive aberrations. In the 45 HNSCC tumours reported here, deletion of $22 \mathrm{q}$ was a relatively infrequent finding occurring in only eight cases; however, the clinical outcome for these patients was particularly poor. Recently, a study of 40 primary oral tumours by quantitative reverse transcriptase-polymerase chain reaction (RT-PCR) identified deletion of the DIA 1 gene, a cytochrome $b 5$ reductase, at 22q13 in $25 \%$ of cases and this loss was also significantly associated with a decrease in survival $(P=0.0018-\log$ rank test; Reis et al, 2002).

One surprising result from this analysis was the low frequency of 11 q13 amplification detected in this cohort of tumours (four out of 45), compared with other studies. To some extent, this may reflect potential dilution of the CGH signal by normal cell contamination as well as the arbitrary discrimination between gain (CGH ratio $1.15-1.5$ ) and amplification (ratio > 1.5). Unlike Bockmühl et al (2000), no association between gain of 11q13 and reduced survival was identified; however, when tumours demonstrating amplification of this region were analysed separately a strong association with reduced disease-free survival was revealed. This association with 11q13 amplification and poor prognosis is consistent with previous classical karyotypic data (Akervall et al, 1995; Meredith et al, 1995).

The demonstration of genetic aberrations exhibiting prognostic significance in this relatively small cohort of tumours, using a lowresolution technology, provides strong encouragement for the continued investigation of the molecular abnormalities underlying HNSCC and other tumours. Such findings demonstrate that molecular characterisation of HNSCC can provide additional markers of prognosis to supplement the classical pathological assessment of tumours. It is important to emphasise that, as with many other HNSCC studies, this cohort consisted of a mixed

Table 3 Chromosomal aberrations demonstrating a significant association with disease-free (upper) and disease-specific survival (lower)

\begin{tabular}{|c|c|c|c|c|c|}
\hline Chromosome & Aberration & Log rank $(P)$ & No. of cases with aberration & \multicolumn{2}{|c|}{ Mean survival (months) } \\
\hline \multicolumn{6}{|l|}{ Disease-free survival } \\
\hline $17 q$ & Gain & 0.049 & 9 & 10.9 & 44.4 \\
\hline $20 \mathrm{q}$ & Gain & 0.001 & 5 & 4.6 & 46.1 \\
\hline $19 p$ & Deletion & 0.002 & II & 13.5 & 44.9 \\
\hline \multicolumn{6}{|l|}{ Disease-specific survival } \\
\hline Nodal status (at surgery) & - & 0.024 & 30 & $(\mathrm{~N}+) 43.9$ & $(\mathrm{~N} 0) 63.9$ \\
\hline $3 q 25-27$ & Gain & 0.043 & 34 & 44.9 & 63.3 \\
\hline $22 q$ & Deletion & 0.011 & 8 & 17.0 & 54.9 \\
\hline
\end{tabular}

$\mathrm{N}+=$ with nodal involvement; $\mathrm{NO}=$ without nodal involvement. 
A

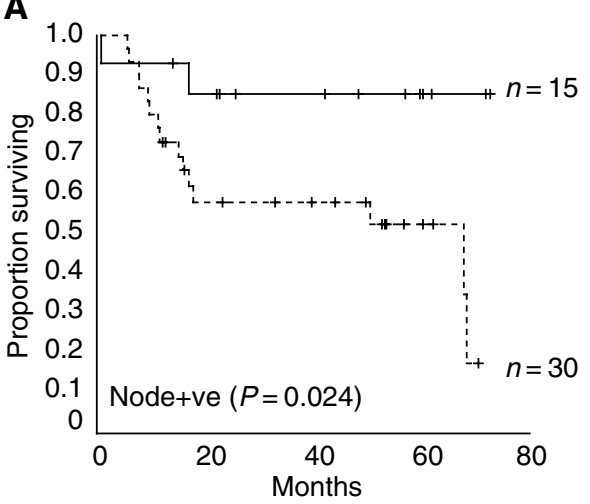

B

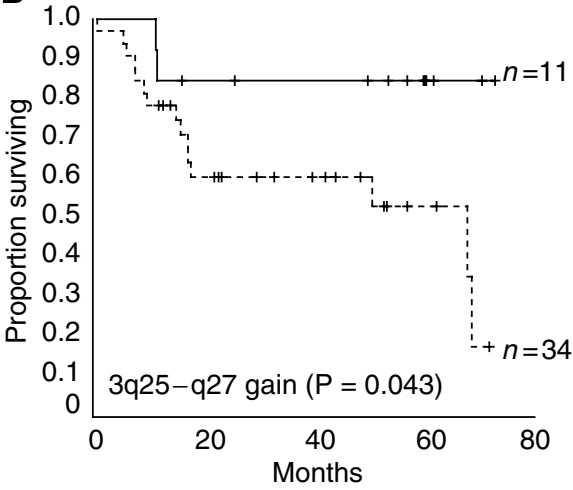

C

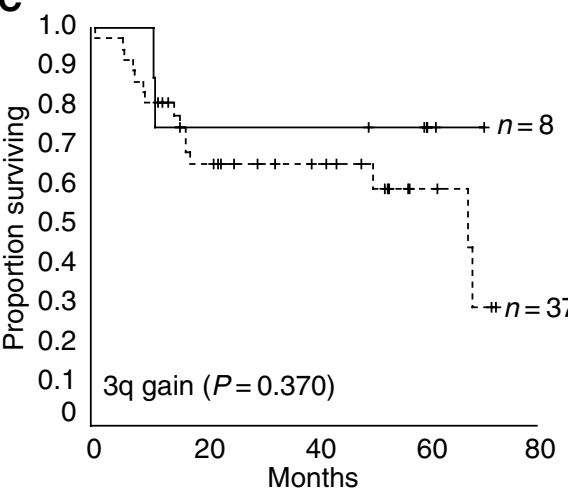

D

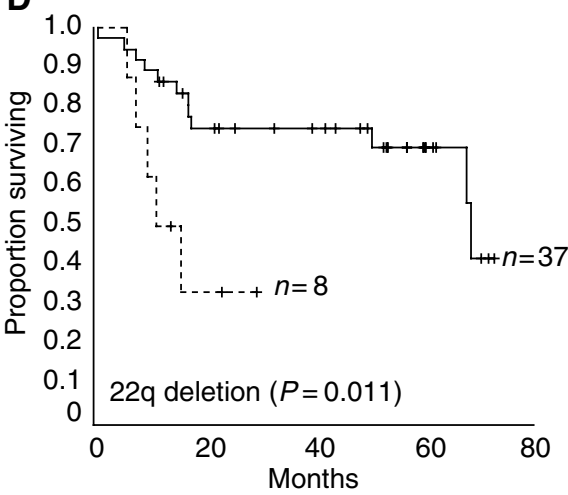

Figure 2 Kaplan-Meier survival curves for disease-specific survival in tumours demonstrating $(\mathbf{A})$ tumours with pathologically proven nodal involvement at time of surgery, (B) gains specifically within the region $3 q 25-q 27$, (C) gains on $3 q$ and (D) deletion of 22q. Dotted lines represent tumours that exhibited the chromosomal imbalance (or nodal involvement in the case of $\mathbf{A}$ ) and solid lines represent those that did not. Crosses indicate censored cases (patients alive at time of analysis).
Table 4 Cox proportional hazard analysis

\begin{tabular}{lcc}
\hline Variable & Cox hazard ratio $\mathbf{( 9 5 \% ~ C L ) ~}$ & $\boldsymbol{P}$-value \\
\hline Nodal status & $4.42(1.00-19.44)$ & 0.049 \\
22q deletion & $3.19(1.04-9.80)$ & 0.042 \\
3q25-27 gain & $3.27(0.73-14.58)$ & 0.121 \\
\hline
\end{tabular}

population of tumour subsites. This fact does not detract from the significance of the findings presented here, but suggests that additional studies on homogeneous populations of HNSCC tumours may reveal additional subsite-specific genetic markers of prognosis, which are masked when analysed as a single entity.

Many of the chromosomal regions identified in this study contain tumour genes already implicated in the tumorigenesis of HNSCC including 3p14 (fhit), 7p12 (EGFR), 7q31 (ING3), 8q24 $(c-m y c), 9 \mathrm{p}(p 16), 11 \mathrm{q} 13$ (ccnd1) and 17p ( $p 53)$. The affected genes in other regions identified in this, and other studies, remain to be fully elucidated. The body of knowledge of genetic aberrations in HNSCC is rapidly growing, and with the advent of DNA microarray technology the copy number and gene expression levels of hundreds of genes can be accurately established. Performing CGH on an ordered array of genomic clones, in place of metaphase chromosomes, dramatically increases the resolution of the technique. This modification of the CGH method has demonstrated copy number changes as low as $100 \mathrm{~kb}$ (SolinasToldo et al, 1997). Such a significant increase in resolution and sensitivity may help resolve discrepancies between CGH studies, that is, regions such as 11q13 that correlate with prognosis in some studies but not others. Microarray-based strategies represent the future of gene copy number analysis in HNSCC and the identification of chromosomal regions with prognostic importance will facilitate the design of such higher resolution strategies, allowing further molecular characterisation of this disease.

\section{ACKNOWLEDGEMENTS}

We gratefully acknowledge Dr Alistair MacDonald of the Department of Histopathology, Hull and East Yorkshire Hospitals for providing pathological assessment of the tumour specimens included in this study and the Applied Statistics Centre, University of Hull, for assistance in the statistical analysis of data.

\section{REFERENCES}

Akervall JA, Jin Y, Wennerberg JP, Zatterstrom UK, Kjellen E, Mertens F, Willen R, Mandahl N, Heim S, Mitelman F (1995) Chromosomal abnormalities involving 11q13 are associated with poor prognosis in patients with squamous cell carcinoma of the head and neck. Cancer 76 (5): $853-859$

Bergamo NA, Rogatto SR, Poli-Frederico RC, Reis PP, Kowalski LP, Zielenska M, Squire JA (2000) Comparative genomic hybridization analysis detects frequent over-representation of DNA sequences at $3 \mathrm{q}$, $7 \mathrm{p}$, and $8 \mathrm{q}$ in head and neck carcinomas. Cancer Genet Cytogenet 119 (1): $48-55$

Bockmühl U, Schluns K, Kuchler I, Petersen S, Petersen I (2000) Genetic imbalances with impact on survival in head and neck cancer patients. Am J Pathol 57 (2): 369-375

Chujo M, Noguchi T, Miura T, Arinaga M, Uchida Y, Tagawa Y (2002) Comparative genomic hybridisation analysis detected frequent overrepresentation of chromosome $3 \mathrm{q}$ in squamous cell carcinoma of the lung. Lung Cancer 38 (1): 23-29

dos Reis PP, Poli-Frederico RC, dos Santos RM, Nishimoto IN, Kowalski LP, Rogatto SR (2002) Distinct regions of loss of heterozygosity on 22q in different sites of head and neck squamous cell carcinomas. Med Sci Monit 8 (3): BR89-BR94 
Hashimoto Y, Oga A, Kawauchi S, Furuya T, Shimizu N, Nakano T, Imate Y, Yamashita H, Sasaki K (2001) Amplification of 3q26 approximately qter correlates with tumor progression in head and neck squamous cell carcinomas. Cancer Genet Cytogenet 129 (1): 52-56

Hermsen M, Guervos MA, Meijer G, Baak J, van Diest P, Marcos CA, Sampedro A (2001) New chromosomal regions with high-level amplifications in squamous cell carcinomas of the larynx and pharynx, identified by comparative genomic hybridisation. J Pathol 194 (2): 177-182

Heselmeyer K, Schrock E, du Manoir S, Blegen H, Shah K, Steinbeck R, Auer G, Ried T (1996) Gain of chromosome 3q defines the transition from severe dysplasia to invasive carcinoma of the uterine cervix. Proc Natl Acad Sci USA 93 (1): 479-484

Jemal A, Murray T, Samuels A, Ghafoor A, Ward E, Thun MJ (2003) Cancer statistics, 2003. CA: Cancer J Clin 53 (1): 5-26

Jeuken JW, Sprenger SH, Wesseling P (2002) Comparative genomic hybridisation: practical guidelines. Diagn Mol Pathol 11 (4): $193-203$

Ma YY, Wei SJ, Lin YC, Lung JC, Chang TC, Whang-Peng J, Liu JM, Yang DM, Yang WK, Shen CY (2000) PIK3CA as an oncogene in cervical cancer. Oncogene 19 (23): 2739-2744

Meredith SD, Levine PA, Burns JA, Gaffey MJ, Boyd JC, Weiss LM, Erickson NL, Williams ME (1995) Chromosome 11q13 amplification in head and neck squamous cell carcinoma. Association with poor prognosis. Arch Otolaryngol Head Neck Surg 21 (7): 790-794

O’Brien CJ, Smith JW, Soong SJ, Urist MM, Maddox WA (1986) Neck dissection with and without radiotherapy: prognostic factors, patterns of recurrence, and survival. Am J Surg 152 (4): 456-463

Redon R, Muller D, Caulee K, Wanherdrick K, Abecassis J, du Manoir S (2001) A simple specific pattern of chromosomal aberrations at early stages of head and neck squamous cell carcinomas: PIK3CA but not p63 gene as a likely target of 3q26-qter gains. Cancer Res 61 (10): 4122-4129

Redon R, Hussenet T, Bour G, Caulee K, Jost B, Muller D, Abecassis J, du Manoir S (2002) Amplicon mapping and transcriptional analysis pinpoint cyclin $\mathrm{L}$ as a candidate oncogene in head and neck cancer. Cancer Res 62 (21): $6211-6217$

Reis PP, Rogatto SR, Kowalski LP, Nishimoto IN, Montovani JC, Corpus G, Squire JA, Kamel-Reid S (2002) Quantitative real-time PCR identifies a critical region of deletion on 22q13 related to prognosis in oral cancer. Oncogene 21 (42): 6480-6487

Shayesteh L, Lu Y, Kuo WL, Baldocchi R, Godfrey T, Collins C, Pinkel D, Powell B, Mills GB, Gray JW (1999) PIK3CA is implicated as an oncogene in ovarian cancer. Nat Genet 21 (1): 99-102

Singh B, Gogineni SK, Sacks PG, Shaha AR, Shah JP, Stoffel A, Rao PH (2001) Molecular cytogenetic characterization of head and neck squamous cell carcinoma and refinement of $3 \mathrm{q}$ amplification. Cancer Res 61 (11): $4506-4513$

Singh B, Stoffel A, Gogineni S, Poluri A, Pfister DG, Shaha AR, Pathak A, Bosl G, Cordon-Cardo C, Shah JP, Rao PH (2002) Amplification of the $3 q 26.3$ locus is associated with progression to invasive cancer and is a negative prognostic factor in head and neck squamous cell carcinomas. Am J Pathol 161 (2): 365-371

Solinas-Toldo S, Lampel S, Stilgenbauer S, Nickolenko J, Benner A, Dohner H, Cremer T, Lichter P (1997) Matrix-based comparative genomic hybridisation: biochips to screen for genomic imbalances. Genes Chromosomes Cancer 20 (4): 399-407

Stafford ND, Ashman JN, MacDonald AW, Ell SR, Monson JR, Greenman J (1999) Genetic analysis of head and neck squamous cell carcinoma and surrounding mucosa. Arch Otolaryngol Head Neck Surg 125 (12): 1341 1348

Struski S, Doco-Fenzy M, Cornillet-Lefebvre P (2002) Compilation of published comparative genomic hybridisation studies. Cancer Genet Cytogenet 135 (1): $63-90$

Volinia S, Hiles I, Ormondroyd E, Nizetic D, Antonacci R, Rocchi M, Waterfield MD (1994) Molecular cloning, cDNA sequence, and chromosomal localization of the human phosphatidylinositol 3-kinase p110 alpha (PIK3CA) gene. Genomics 24 (3): 472-477 